\title{
Comparison of Percutaneous and Surgical Right Ventricular Assist Device Support Following Durable Left Ventricular Assist Device Insertion
}

\author{
Ellie J Coromilas, MD², Koji Takeda, MD PhD², Masahiko Ando, MD², Marisa Cevasco, MD², \\ Phillip Green, MD, MS ${ }^{1}$, Dimitri Karmpaliotis, MD, PhD ${ }^{1}$, Ajay Kirtane, MD, SM${ }^{1}$, Veli K \\ Topkara, MD, MS ${ }^{1}$, Melana Yuzefpolskaya, MD1', Hiroo Takayama, MD, PhD², Yoshifumi \\ Naka, MD PhD ${ }^{2}$, Daniel Burkhoff, MD PhD ${ }^{1}$, Paolo C. Colombo, MD ${ }^{1}$, and A. Reshad Garan, \\ MD1 \\ ${ }^{1}$ Division of Cardiology, Department of Medicine, Columbia University Medical Center - New York \\ Presbyterian, New York, NY \\ 2 Division of Cardiothoracic Surgery, Department of Surgery, Columbia University Medical Center \\ - New York Presbyterian, New York, NY
}

\section{Abstract}

Background: Early right ventricular (RV) failure after LVAD increases morbidity and mortality. Percutaneous RVAD (pRVAD) support is an alternative to more invasive surgical RVAD (sRVAD).

\begin{abstract}
Methods: We retrospectively reviewed patients receiving isolated pRVAD or SRVAD after durable LVAD at our center between 2007-2018. Hemodynamic parameters before and after implantation and survival outcomes were compared among groups.
\end{abstract}

Results: Nineteen patients received pRVAD and 21 received sRVAD. Hemodynamic parameters improved immediately with pRVAD; central venous pressure decreased (15.9 \pm 2.4 to $12.3 \pm 3.2$ $\mathrm{mmHg}, \mathrm{p}<0.001)$ and cardiac index increased $\left(2.4 \pm 0.5\right.$ to $\left.3.5 \pm 0.8 \mathrm{~L} / \mathrm{min} / \mathrm{m}^{2}, \mathrm{p}<0.001\right)$. These were sustained following device removal and were similar to those with sRVAD. Patients with pRVAD required fewer blood transfusions and mechanically-ventilated days than with SRVAD. Among survivors, ICU and hospital days were fewer with pRVAD [21(16-27) vs. 34(27-46) ICU days, $\mathrm{p}=0.01 ; 43.5(30-66)$ vs. $91(62-111)$ hospital days, $\mathrm{p}=0.03]$. There was no significant difference in 30-day mortality with pRVAD compared to sRVAD ( $21.1 \%$ vs. $42.9 \%$, $\mathrm{p}=0.14)$, but there was a trend toward higher rate of discharge free from hemodialysis $(73.7 \%$ vs. $47.6 \%$, $\mathrm{p}=0.09$ ).

Conclusions: Novel pRVAD systems for RV failure provide hemodynamic benefits comparable to SRVAD, are associated with less morbidity, and should be considered an alternative to sRVAD.

Corresponding Author: A. Reshad Garan, M.D., Assistant Professor of Medicine, Center for Advanced Cardiac Care, Columbia University Medical Center - New York Presbyterian, 622 West 168th St, PH9-977, Phone: (212) 305-4600, Fax: (212) 305-7439, arg2024@cumc.columbia.edu.

Publisher's Disclaimer: This is a PDF file of an unedited manuscript that has been accepted for publication. As a service to our customers we are providing this early version of the manuscript. The manuscript will undergo copyediting, typesetting, and review of the resulting proof before it is published in its final citable form. Please note that during the production process errors may be discovered which could affect the content, and all legal disclaimers that apply to the journal pertain. 


\section{Keywords}

Right ventricular failure; Right ventricular assist device; Percutaneous; Left ventricular assist device; Heart failure

\section{INTRODUCTION}

Early right ventricular (RV) failure affects up to $30-40 \%$ of patients undergoing left ventricular assist device (LVAD) insertion, and is associated with increased morbidity and mortality in this population. ${ }^{1-5}$ Multiple tools for identifying patients at risk of RV failure have been proposed with varying degrees of accuracy. ${ }^{2-4,6}$ While some patients are supported sufficiently with medical therapy, others require RV mechanical support, traditionally with surgically implanted devices.

Since early RV dysfunction often improves sufficiently over a short period of time, many patients may not require prolonged RV mechanical support. ${ }^{7}$ Typically, recovery occurs within days to weeks, making temporary mechanical support with less invasive modalities an attractive alternative to surgically placed devices in this population as a bridge to recovery.

As an alternative to surgical right ventricular assist device (sRVAD), recent advances in percutaneous technology have brought multiple devices into practice that allow rapid deployment of RV mechanical support percutaneously. ${ }^{7-11}$ These devices include the Impella RP (Abiomed, Danvers, MA), a micro-axial flow intra-corporeal device, and the Protek Duo (TandemLife, Pittsburgh, PA) dual lumen cannula which can be used with a centrifugal flow extra-corporeal pump.

Currently, data regarding the efficacy of support with such percutaneous devices are limited. In the present study, we sought to characterize the hemodynamic effects and outcomes observed with percutaneous RVADs (pRVAD) and compare them to those associated with sRVADs.

\section{METHODS}

\section{Patient Population}

We retrospectively reviewed all patients who underwent isolated pRVAD or sRVAD implantation at Columbia University after durable LVAD insertion between March, 2007 and April, 2018. We included adult patients who received an Impella RP or a Protek Duo with either a TandemHeart (TandemLife, Pittsburgh, PA) or Centrimag (Thoratec, Pleasanton, CA) pump and those who had placement of a surgical RVAD (Centrimag) for refractory RV failure following LVAD implantation. RV failure was defined according to our institutional definition as a cardiac index $(\mathrm{CI})<2.2 \mathrm{~L} / \mathrm{min} / \mathrm{m}^{2}$ with a mean arterial pressure (MAP) $<$ $70 \mathrm{mmHg}$ or the need for more than 2 high dose vasoactive medications to maintain these parameters despite an elevated central venous pressure (CVP). Patients who had an RVAD placed for other indications (e.g. acute myocardial infarction, post-cardiotomy shock), or those who had any RVAD implanted concomitantly with the LVAD were excluded from the analysis. Two patients who received both pRVAD and sRVAD were excluded from the 
analysis (one received sRVAD first, one received pRVAD first). This study was approved by the Columbia University Institutional Review Board.

\section{Variables and Outcomes of Interest}

All clinical data were collected through a review of electronic medical records.

Demographic and clinical information were collected including comorbidities and laboratory values. Hemodynamic data including LVAD flows were collected prior to RVAD insertion, 12 and 24 hours after device insertion, and 24 hours following device removal. Cardiac output (CO) and $\mathrm{CI}$ were calculated using the Fick method. Other hemodynamic values were calculated to evaluate baseline ventricular function including cardiac power output, cardiac power index, pulmonary artery pulsatility index (PAPI), and RV stroke work index (RVSWI).

The primary outcomes of interest were survival at 30 days and survival to hospital discharge. Secondary outcomes collected included ICU and hospital length of stay which were calculated both for all patients and for patients who survived to hospital discharge. Clinical outcome measures during device support were collected, including days on mechanical ventilation, hours of inhaled nitric oxide therapy, transfusions of packed red blood cells, and need for renal replacement therapy with continuous veno-venous hemofiltration or intermittent hemodialysis. Of note, for patients who underwent orthotopic heart transplantation during the index admission, length of stay outcomes were censored at time of transplantation.

\section{Statistical Analysis}

For continuous variables, normality was tested with the Shapiro-Wilk test, and data are presented as means \pm standard deviation or medians with interquartile range where appropriate. Categorical variables are presented as counts and percentages. Student's t-test and Wilcoxon Rank Sum test were used where appropriate to compare continuous variables across groups and the Fisher's Exact and Chi-squared tests were used where appropriate to compare categorical variables across groups. Significance was defined as $\mathrm{p}<0.05$.

\section{RESULTS}

We identified 19 patients who received pRVAD and 21 patients who received SRVAD for RV failure following LVAD implantation. During this time period, 30 patients underwent surgical RVAD insertion at the same time as durable LVAD insertion. Patients received sRVAD throughout the study period, however pRVADs were only used between 2015 and 2018. The types of RVAD insertion by implant year are displayed in Figure 1. Patients had a mean age of $58.6 \pm 11.8$ years and the majority were men. Clinical and demographic characteristics of this population, including significant comorbidities are shown in Table 1. There was no significant difference in demographic parameters, comorbidities, or common laboratory values prior to device implantation among patients receiving percutaneous or surgical devices.

Prior to LVAD implantation, the mean right atrial/pulmonary capillary wedge (RA/PCW) ratio was $0.43 \pm 0.19$, the mean RVSWI was $7.1 \pm 4.3 \mathrm{~g} / \mathrm{m}^{2} /$ beat, and the mean PAPI was 4.0 
\pm 4.6. Six (15\%) patients had a RA/PCW greater than 0.63 and $11(27.5 \%)$ had a PAPI less than 1.85 . Three (7.5\%) patients were at high risk of RV failure according to the RV Failure Risk Score ${ }^{4}$ and no (0\%) patients were at high risk according to the EUROMACS RV failure risk score. ${ }^{2}$

Hemodynamics measured after durable LVAD implantation at the time of RVAD implantation were similar between the two groups and reflect a population with significant hemodynamic compromise (Table 2). Prior to RVAD implantation, patients in the combined cohort had a mean CVP of $15.9 \pm 2.8 \mathrm{mmHg}$ with diastolic pulmonary artery pressure of $22.3 \pm 5.1 \mathrm{mmHg}$, mean pulmonary arterial pressure of $28.3 \pm 6.9 \mathrm{mmHg}$, and pulmonary vascular resistance of $2.3 \pm 1.4$ Wood units. Mean arterial pressure (MAP) was $73.8 \pm 11.8$ $\mathrm{mmHg}$ while on an average of $3.7 \pm 1.0$ vasopressors and inotropes, with average systemic vascular resistance (SVR) was $1006 \pm 506$ dyne* $\mathrm{sec} / \mathrm{cm}^{5}$. Mean CO and CI prior to RVAD implantation was $5.0 \pm 2.0 \mathrm{~L} / \mathrm{min}$ and $2.4 \pm 0.8 \mathrm{~L} / \mathrm{min} / \mathrm{m}^{2}$, respectively, with cardiac power index of $0.4 \pm 0.2 \mathrm{Watt} / \mathrm{m}^{2}$. The average PAPI was $1.2 \pm 0.6$ and RVSWI was $3.4 \pm 2.2 \mathrm{~g} / \mathrm{m}^{2} /$ beat, consistent with severe RV dysfunction.

Of the 19 patients receiving pRVAD, 4 (21.1\%) received Impella RP and 15 (78.9\%) received Protek Duo cannulation (Table 1; Figure 2). In 12 instances, the Protek Duo was used with the TandemHeart pump and in the remaining 3 cases, a Centrimag pump was utilized with this cannula. All 21 patients supported with sRVAD had Centrimag RVAD. Eighteen $(85.7 \%)$ had right atrial to pulmonary artery (PA) cannulation, and $3(14.3 \%)$ had RV to PA cannulation. Patients underwent RVAD implantation a median of 1 day (IQR 14.5) following LVAD implantation; the median time to sRVAD implantation was 3 days (IQR $1-7$ ) and to pRVAD was 1 day (IQR $1-3 ; \mathrm{p}=0.15$ ). RVAD flow was significantly higher with sRVAD compared to pRVAD (5.2 \pm 0.9 vs. $4.0 \pm 0.4 \mathrm{~L} / \mathrm{min}, \mathrm{p}<0.001)$; LVAD flow while on RVAD support was higher in the surgical group compared to percutaneous $(5.8 \pm 1.7$ vs. $4.8 \pm 0.8 \mathrm{~L} / \mathrm{min}, \mathrm{p}=0.02)$. Table 3 summarizes hemodynamic changes seen with device support and after device removal with pRVAD and sRVAD. Hemodynamic parameters including CVP, MAP, and CI improved immediately with pRVAD(Figure 3). These changes were accompanied by a substantial reduction in vasopressor doses (Figure 4). The hemodynamic changes with sRVAD and pRVAD were similar (Figure 4). There was a similar sustained decrease in CVP seen with both devices, with similar values at baseline $(16.2 \pm 3.3$ vs. $15.6 \pm 2.4 \mathrm{mmHg}, \mathrm{p}=0.56), 24$ hours following device insertion $(11.2 \pm 2.8$ vs. $12.3 \pm 3.2 \mathrm{mmHg}, \mathrm{p}=0.29)$, and after device removal $(11.2 \pm 4.2$ vs. $10.8 \pm 2.9 \mathrm{mmHg}$, $\mathrm{p}=0.88$ ). $\mathrm{CO}$ and $\mathrm{CI}$ were similar at most time-points on RVAD support. CI was higher 24 hours following device insertion with sRVAD compared to PRVAD ( $4.6 \pm 1.3$ vs. $3.5 \pm 0.8$ $\mathrm{L} / \mathrm{min} / \mathrm{m}^{2}, \mathrm{p}=0.01$ ), however this difference was not sustained after device removal ( 3.5 \pm 0.6 vs. $3.3 \pm 0.6 \mathrm{~L} / \mathrm{min} / \mathrm{m}^{2}, \mathrm{p}=0.45$ ).

Figure 4 illustrates changes in vasopressor and inotrope requirements with both devices, as well as change in urine output. There was a significant decrease in norepinephrine and vasopressin dosages with both pRVAD and sRVAD support; among patients supported with pRVAD, norepinephrine dose decreased from $13.2 \pm 7.0$ to $6.3 \pm 5.0 \mathrm{mcg} / \mathrm{min} 24$ hours after device insertion ( $\mathrm{p}=0.004)$ and to $3.7 \pm 4.4 \mathrm{mcg} / \mathrm{min}$ at 48 hours $(\mathrm{p}<0.001)$. Vasopressin dose decreased from $4.6 \pm 1.5$ to $3.2 \pm 2.1 \mathrm{U} / \mathrm{hr}$ at 24 hours ( $\mathrm{p}=0.04$ ) and $2.0 \pm 1.8 \mathrm{U} / \mathrm{hr}$ at $48 \mathrm{hr}$ 
$(\mathrm{p}<0.001)$. There was no significant change in inotropes at 48 hours with either device, though there was a non-significant decrease in epinephrine doses with pRVAD and in epinephrine and dobutamine doses with sRVAD.

Indicators of end organ function were similar with pRVAD and SRVAD support, with similar serum lactate levels, creatinine, and urine output between the two groups (Table 3). Serum lactate was reduced 24 hours after pRVAD insertion, though this difference was not statistically significant $(2.28 \pm 1.4$ vs. $1.60 \pm 1.53 \mathrm{mg} / \mathrm{dL}, \mathrm{p}=0.13)$. There was no significant change in renal function with pRVAD support as measured by serum creatinine $(1.63 \pm 0.6$ vs. $1.78 \pm 1.0 \mathrm{mg} / \mathrm{dL}, \mathrm{p}=0.56$ ), though there was a trend towards an increase in urine output $(1700 \pm 1145$ vs. $3081 \pm 3017 \mathrm{~mL} / 24 \mathrm{hr}, \mathrm{p}=0.07)$ at 48 hours (Figure $4 \mathrm{C})$.

Patients who received pRVAD had a median time on the device of 9 days (IQR 7-13) while those receiving SRVAD had the device for 18 days (IQR 11-28); when controlling for patients undergoing transplantation or dying on RVAD these were not significantly different $(\mathrm{p}=0.57)$. Patients supported with $\mathrm{pRVAD}$ required significantly less time mechanically ventilated than those supported with pRVAD (8.5 [IQR 2-19] vs. 21 [IQR 10-32 days, $\mathrm{p}=0.03$ ). There was no difference in use of inhaled nitric oxide (54 [IQR 30-161] vs. 54.5 [IQR 2.5-121.5] days, $\mathrm{p}=0.75$ ), or need for temporary renal replacement therapy (33.3\% vs. $42.8 \%, \mathrm{p}=0.52$ ). Patients undergoing pRVAD support required significantly fewer transfusions of PRBC than those with SRVAD (3 [IQR 0-6] vs. 6.5 [IQR 5-16), p<0.001). Three patients (14.3\%) receiving sRVAD ultimately had the device explanted at the time of orthotopic heart transplantation; one patient (5.3\%) with pRVAD was bridged to transplantation while on RVAD device support.

Peak lactate dehydrogenase (LDH) while on RVAD support was $1006 \pm 660 \mathrm{U} / \mathrm{L} 1207$ $\pm 1242 \mathrm{U} / \mathrm{L}$ in the percutaneous and surgical cohorts, respectively ( $\mathrm{p}=0.53$ ). No device was removed due to hemolysis. For $1(8.3 \%)$ patient with Protek Duo pRVAD, a TandemHeart pump was changed to a Centrimag pump due to pump malfunction (low device line pressure) and for 1 (33.3\%) patient, the Impella RP device fractured upon removal from the body, though all components of the device were removed percutaneously without incident. Additionally, 1 (33.3\%) patient with Impella RP pRVAD and 1 (6.7\%) with Protek Duo required return to the cardiac catheterization laboratory for repositioning of the pRVAD. Eight $(38.1 \%)$ patients supported by sRVAD required return to the operating room for mediastinal exploration in the setting of bleeding while on SRVAD support.

Patients receiving pRVAD required a median of 21 (10-27) ICU days following implantation, compared to 27 days (IQR 15-44, $\mathrm{p}=0.14$ ) for those receiving sRVAD. Among those surviving to hospital discharge, ICU length of stay was 21 days (IQR 16-27) for patients receiving pRVAD and 34 days (IQR 27-46) for those receiving $\operatorname{sRVAD}(\mathrm{p}=0.01)$. Post-RVAD hospital length of stay was 34.5 days (IQR 21-59) in the group receiving pRVAD and 60 days (IQR 21-95) in the group receiving sRVAD ( $\mathrm{p}=0.35)$. Among survivors, hospital length of stay was significantly shorter for patients receiving pRVAD (43.5 [IQR 30-66] vs. 91 [IQR 62-111] days, p=0.03). 
Mortality with pRVAD was lower when compared to that with sRVAD though this difference was not statistically significant. Three patients (15.8\%) with pRVAD died within 30 days of device implantation, compared with $7(33.3 \%)$ patients with $\operatorname{RRVAD}(\mathrm{p}=0.29)$. Fifteen (78.9\%) patients with pRVAD and 11 (52.4\%) with sRVAD survived to discharge (risk difference $=0.22[95 \%$ confidence interval $-0.06-0.50]$ and OR $2.81[95 \%$ confidence interval $0.69-11.42$ ], $\mathrm{p}=0.14)$. There was a trend towards a higher rate of discharge free from dialysis among those treated with pRVAD $(73.7 \%$ vs. $47.6 \%, p=0.09$; table 4$)$. Oneand two-year survival was $52.4 \%$ and $38.1 \%$ for those treated with sRVAD and $78.9 \%$ and $70.2 \%$ for those with pRVAD $(\mathrm{p}=0.08)$.

\section{DISCUSSION}

Our data provide a comparison between sRVAD and two more recent percutaneous options for RVAD support following durable LVAD implantation. Our principal findings are as follows: 1) both SRVAD and pRVAD support systems provided immediate improvement in hemodynamic profiles for patients, 2) while sRVAD provided higher overall flow than pRVAD the hemodynamic improvements were similar between these two types of device, and 3) pRVAD use was associated with less morbidity than sRVAD use. Our goal was not to compare the two percutaneous devices studied here, but rather to compare the percutaneous approach to the more traditional surgical intervention.

In our cohort of patients with severe RV failure after LVAD implantation, pRVAD and sRVAD support both provided immediate improvement in hemodynamic parameters. Despite pRVAD support resulting in lower flow compared to sRVAD support, hemodynamics were comparable with the two devices. Specifically, we observed a significant reduction in CVP and improvement in cardiac output as well as an improvement in MAP with rapid decrease in vasopressor dose requirement seen within hours of pRVAD placement. Not surprisingly, this was coupled with a doubling of the daily urine output, though this was not statistically significant $(\mathrm{p}=0.07)$.

Importantly, in the majority of patients, the RV was able to recover from the various insults experienced in the post-operative period so that the device could be removed. While the mean SVR was within normal limits for the study cohort, it is important to understand that this was while patients were receiving multiple vasopressors at high doses. This underscores the fact that the typical hemodynamic profile of RV failure after LVAD implantation is one of a vasoplegic patient with a relatively low cardiac index (despite LVAD and multiple inotropes). While the RV will typically be able to eventually wean from support, when faced with vasodilatory physiology it is unable to provide that increase in cardiac output that would compensate for the reduction in SVR. In combination, these two hemodynamic derangements can be very hard to overcome with vasoactive medications alone. For this reason, easily deployed circulatory support devices designed to support the RV and spare the patient's end-organs from the effects of high dose vasoconstrictors have the potential to significantly improve the recovery from LVAD implantation for those who transiently manifest this pathophysiology. 
Several authors have previously described the use of percutaneous or minimally invasive

RVADs. Kapur et al. reported the first study using percutaneous mechanical RV support with the TandemHeart device in 9 patients, though the majority had suffered an inferior myocardial infarction, as opposed to our cohort of RV failure following LVAD implantation. ${ }^{9}$ Haneya et al. described the use of a minimally invasive surgical RVAD in 8 patients with RV failure following LVAD implantation, and found similar improvement in hemodynamic parameters to those seen in our cohort. ${ }^{8}$ These authors described the insertion of the outflow cannula through a Dacron graft anastomosed to the PA meaning that RVAD deployment was more invasive but that removal could be performed with minimal intervention when patients were weaned from RVAD support. Anderson et al. published the largest study examining the use of percutaneous RV support in a prospective, single-arm trial using the Impella RP. ${ }^{7}$ This study included 30 patients with RV failure, 18 of whom required RV support after LVAD implantation. These authors also demonstrated improvement in hemodynamics similar in magnitude to those in our cohort. This study did not have a comparator group but instead compared outcomes to a benchmark established for a contemporary sRVAD. Most recently, Ravichandran et al. described the use of the Protek Duo catheter to provide pRVAD support following LVAD implantation. ${ }^{11}$ However, our data represent the largest comparison of hemodynamic changes and outcomes between the two device support strategies.

In our cohort of early RV failure patients, support with pRVAD was associated with less morbidity compared with sRVAD support. Specifically, those supported with pRVAD had a shorter period of mechanical ventilation, as well as less requirement for blood transfusion. There was a trend towards improved discharge free from dialysis among the pRVAD group. Additionally, hospital and ICU length of stay after RVAD insertion among survivors was shorter with the percutaneous devices. This decreased morbidity is likely related to multiple factors. First, the pRVAD approach is less invasive, allowing for more rapid recovery from both the implantation and the removal procedure. Second, though not significant, the median time to pRVAD implantation following LVAD was one third the time to sRVAD placement. In addition, serum creatinine, transaminases, and lactate were numerically lower at baseline (though not statistically significant) in patients receiving pRVAD. Though the difference in time to RVAD implantation was not significantly different, these findings raise the possibility that the ability to rapidly deploy the pRVAD in the cardiac catheterization laboratory may lower the threshold for initiating RV support and, in turn, result in overall improved outcomes.

Planned RVAD support for patients at high risk of RV failure likely remains the optimal strategy when implanting a durable LVAD. ${ }^{12}$ Similarly, even when RVAD implantation is unplanned, patients with overt RV failure manifesting in the operating room are likely better supported by concomitant RVAD implantation as opposed to delayed implantation if the patient's condition does not improve. ${ }^{13}$ However, the fact that most of the patients in this cohort were not deemed high risk for RV failure underscores the limitations of the currently available risk models and highlights the need for a rapidly deployed RV support device. Therefore, for RV failure manifesting unexpectedly after LVAD implantation, our data suggest that percutaneous support may be a viable alternative to a surgical support device in many cases. 


\section{Limitations}

It is important to note that this is a single center, retrospective study, which introduces several important limitations. Because patients were not randomized to SRVAD or pRVAD, there may have been selection bias in their use; furthermore, as both pRVAD systems used are relatively novel, the two cohorts are not entirely contemporary and device use changed over time. It is possible that the risk of RV failure varied with growing experience managing LVAD patients and this may have contributed to differences between the cohorts of patients receiving the different types of RVAD. Importantly though, we did not note differences in demographic or baseline hemodynamic characteristics between those receiving SRVAD and pRVAD. While we limited our analysis to patients who received continuous-flow LVADs, the risk of RV failure may vary with different pump technology. Additionally, because hemodynamic data were collected retrospectively, full hemodynamic profiles were not available for all patients. The relatively small number of patients in our study limited our ability to detect differences in outcomes between the devices studied, though to our knowledge this is the largest comparison of these two device strategies. Finally, in order to better understand the differences between these two devices, we limited our analysis to patients with RV failure after LVAD implantation, thus limiting our ability to extrapolate these conclusions to other etiologies of RV failure.

\section{Conclusion}

Novel percutaneous RVAD support systems allow for rapid deployment of RV mechanical support following durable LVAD implantation. These systems provide almost immediate improvement in hemodynamics and are associated with less morbidity than their surgical counterparts, and should be considered viable alternatives to SRVAD placement for this patient population.

\section{Acknowledgments}

The authors would like to acknowledge Jimmy Duong, MPH for his assistance. This publication was supported by the National Center for Advancing Translational Sciences, National Institutes of Health, through Grant Number UL1TR001873. The content is solely the responsibility of the authors and does not necessarily represent the official views of the NIH. Dr. Kirtane reports institutional funding to Columbia University and/or Cardiovascular Research Foundation from Medtronic, Boston Scientific, Abbott Vascular, Abiomed, CSI, CathWorks, Siemens, Philips, ReCor Medical. Dr. Naka has received consulting fees from St. Jude Medical (St. Paul, MN). Dr. Burkhoff reports institutional funding to Columbia University and/or Cardiovascular Research Foundation from Abiomed (Danvers, MA). Dr. Garan is supported by National Institutes of Health Grant No. KL2TR001874, has previously received honoraria from Abiomed (Danvers, MA) and is now an unpaid advisor for Abiomed. None of these entities had a role in the preparation of this manuscript. All other authors have reported that they have no relationships relevant to the contents of this manuscript to disclose.

\section{Abbreviations}

RV

LVAD

RVAD

CI right ventricle

left ventricular assist device

right ventricular assist device

cardiac index 


$\begin{array}{ll}\text { CO } & \text { cardiac output } \\ \text { MAP } & \text { mean arterial pressure } \\ \text { CVP } & \text { central venous pressure } \\ \text { RVSWI } & \text { right ventricular stroke work index } \\ \text { PAPI } & \text { pulmonary artery pulsatility index } \\ \text { SVR } & \text { systemic vascular resistance }\end{array}$

\section{REFERENCES}

1. Kiernan MS, Grandin EW, Brinkley M, Jr, et al. Early Right Ventricular Assist Device Use in Patients Undergoing Continuous-Flow Left Ventricular Assist Device Implantation: Incidence and Risk Factors From the Interagency Registry for Mechanically Assisted Circulatory Support. Circ Heart Fail. 2017;10.

2. Soliman OII, Akin S, Muslem R, et al. Derivation and Validation of a Novel RightSided Heart Failure Model After Implantation of Continuous Flow Left Ventricular Assist Devices: The EUROMACS (European Registry for Patients with Mechanical Circulatory Support) Right-Sided Heart Failure Risk Score. Circulation. 2018;137:891-906.3. [PubMed: 28847897]

3. Kormos RL, Teuteberg JJ, Pagani FD et al. Right ventricular failure in patients with the HeartMate II continuous-flow left ventricular assist device: incidence, risk factors, and effect on outcomes. J Thorac Cardiovasc Surg 2010;139:1316-24. [PubMed: 20132950]

4. Matthews JC, Koelling TM, Pagani FD, Aaronson KD. The right ventricular failure risk score a preoperative tool for assessing the risk of right ventricular failure in left ventricular assist device candidates. J Am Coll Cardiol 2008;51:2163-72. [PubMed: 18510965]

5. Konstam MA, Kiernan MS, Bernstein D et al. Evaluation and Management of Right-Sided Heart Failure: A Scientific Statement From the American Heart Association. Circulation 2018.

6. Morine KJ, Kiernan MS, Pham DT, Paruchuri V, Denofrio D, Kapur NK. Pulmonary Artery Pulsatility Index Is Associated With Right Ventricular Failure After Left Ventricular Assist Device Surgery. J Card Fail. 2016;22:110-6. [PubMed: 26564619]

7. Anderson MB, Goldstein J, Milano C et al. Benefits of a novel percutaneous ventricular assist device for right heart failure: The prospective RECOVER RIGHT study of the Impella RP device. J Heart Lung Transplant 2015;34:1549-60. [PubMed: 26681124]

8. Haneya A, Philipp A, Puehler T et al. Temporary percutaneous right ventricular support using a centrifugal pump in patients with postoperative acute refractory right ventricular failure after left ventricular assist device implantation. Eur J Cardiothorac Surg 2012;41:219-23. [PubMed: 21641814]

9. Kapur NK, Paruchuri V, Korabathina R et al. Effects of a percutaneous mechanical circulatory support device for medically refractory right ventricular failure. J Heart Lung Transplant 2011;30:1360-7. [PubMed: 21868253]

10. Takayama H, Naka Y, Kodali SK et al. A novel approach to percutaneous right-ventricular mechanical support. Eur J Cardiothorac Surg 2012;41:423-6. [PubMed: 21737292]

11. Ravichandran AK, Baran DA, Stelling K, Cowger JA, Salerno CT. Outcomes withthe Tandem Protek Duo Dual-Lumen Percutaneous Right Ventricular Assist Device. ASAIO J 2017.

12. Fitzpatrick JR, 3rd, Frederick JR, Hiesinger W, et al. Early planned institution of biventricular mechanical circulatory support results in improved outcomes compared with delayed conversion of a left ventricular assist device to a biventricular assist device. J Thorac Cardiovasc Surg. 2009;137:971-7. [PubMed: 19327526]

13. Takeda K, Naka Y, Yang JA, et al. Outcome of unplanned right ventricular assist device support for severe right heart failure after implantable left ventricular assist device insertion. J Heart Lung Transplant. 2014;33:141-8. [PubMed: 23932442] 


\section{Highlights}

Both surgical and percutaneous RVAD provided immediate improvement in hemodynamic profiles for patients. While surgical RVAD provided higher overall flow than percutaneous the hemodynamic improvements were similar between these two types of device. Finally, percutaneous RVAD use was associated with less morbidity than surgical devices. 
Type of Right Ventricular Assist Device By Implant Year

18

16

14

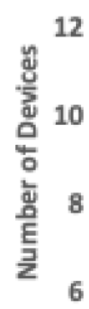

4

2

0

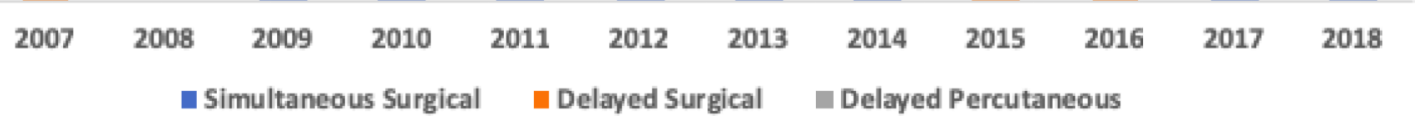

Figure 1.

Type of Right Ventricular Assist Device by Implant Year. 
A.

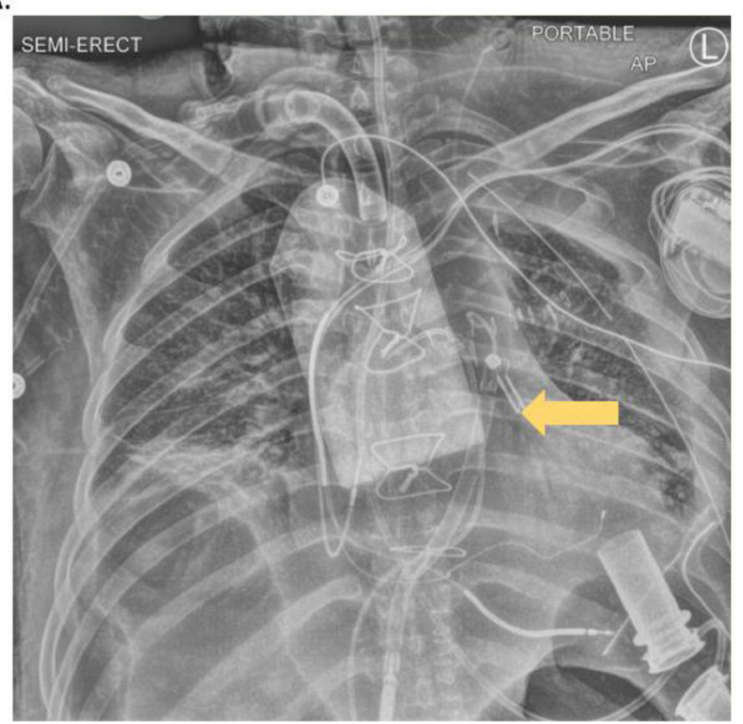

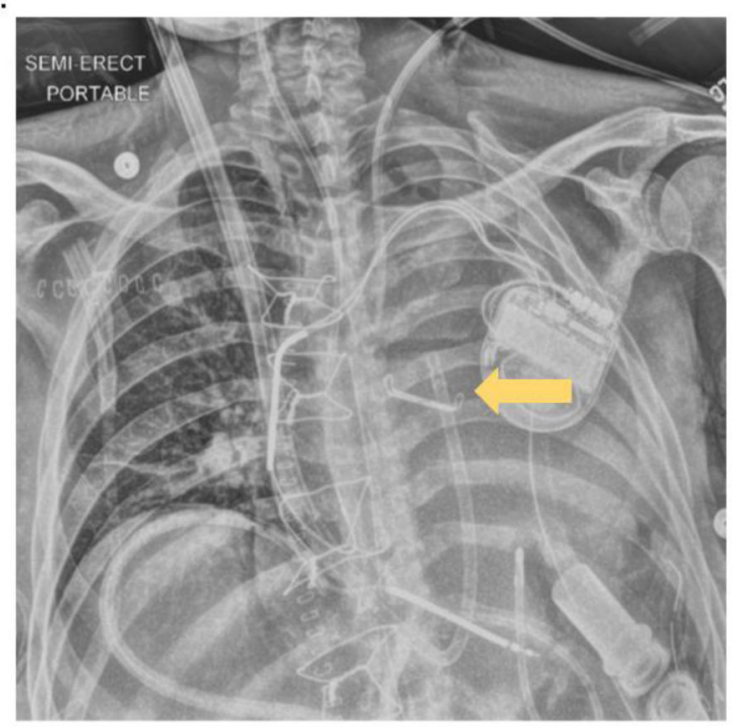

Figure 2.

Chest radiographs illustrating placement of A) impella RP with femoral cannulation and B) Protek Duo with internal jugular cannulation 
A.

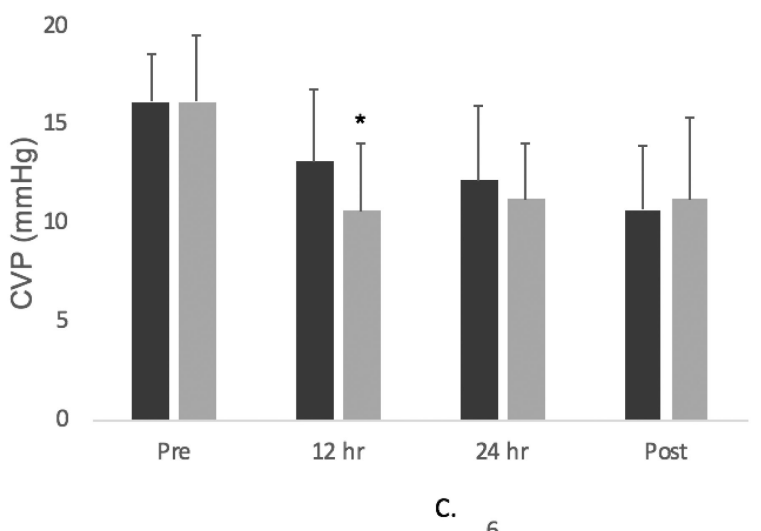

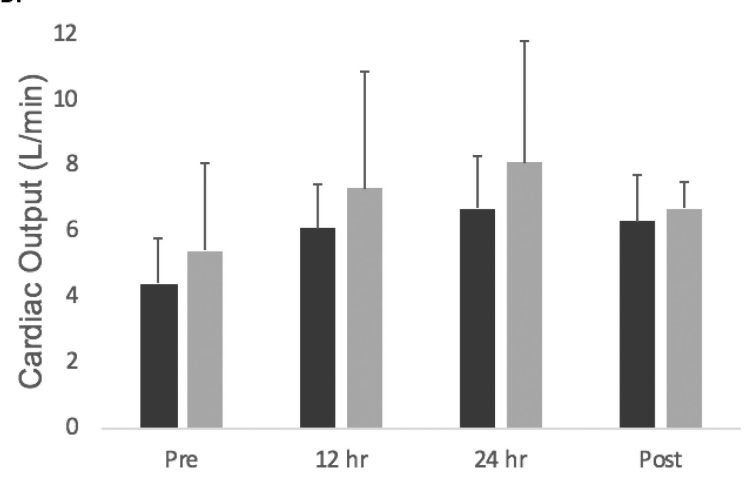

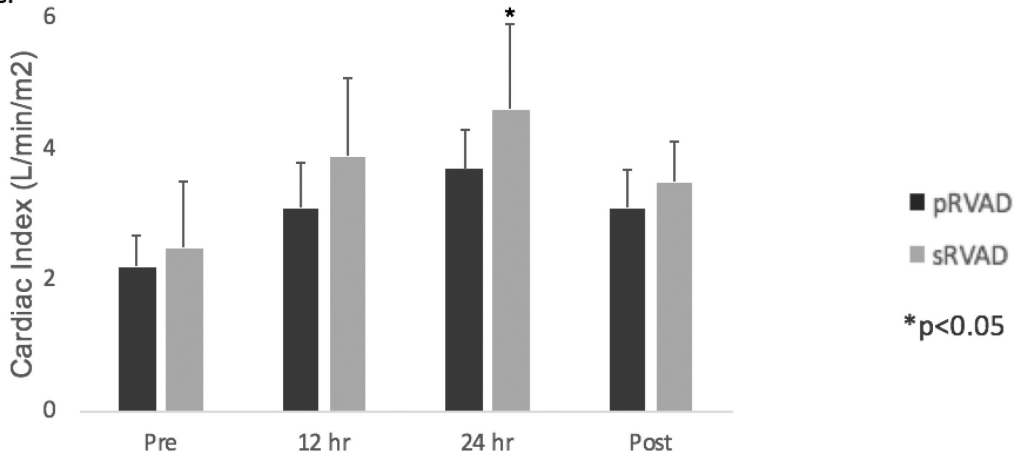

Figure 3.

Changes in hemodynamic parameters with pRVAD and sRVAD support 
A.

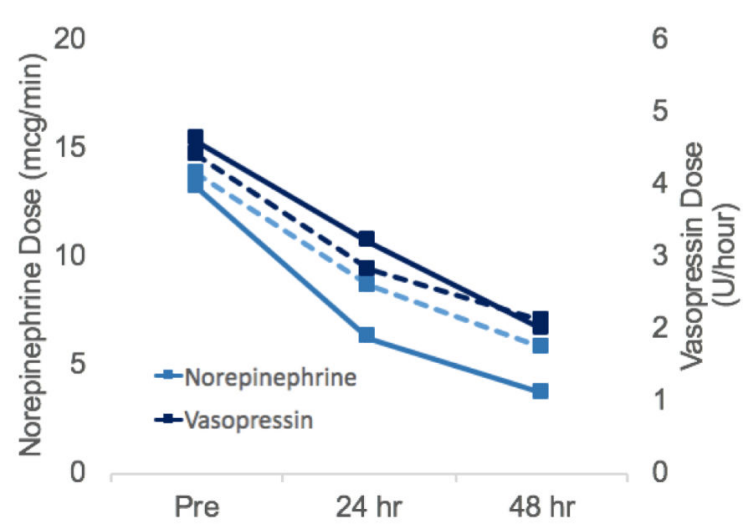

B.

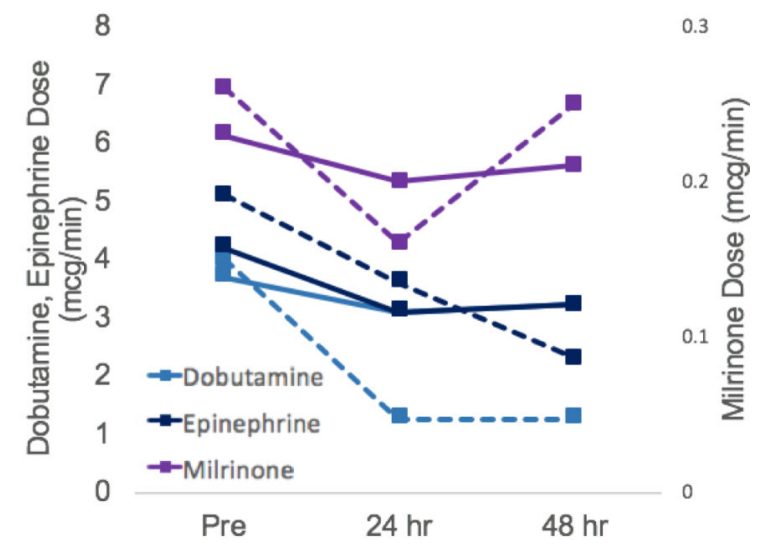

C. 4000

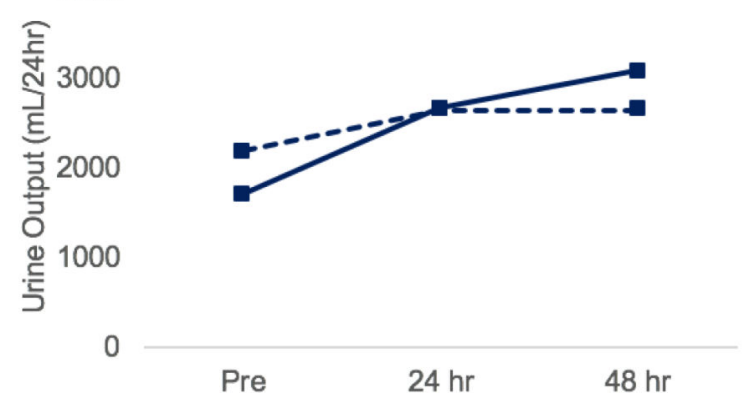

Figure 4.

Changes in A) vasopressor and B) inotrope doses over time with pRVAD (indicated by solid line) and sRVAD (indicated by dotted line) 
Table 1.

Demographics and baseline characteristics of patients receiving percutaneous and surgical RVAD. pRVAD, percutaneous right ventricular assist device; sRVAD, surgical right ventricular assist device. BMI, body mass index; CAD, coronary artery disease; HTN, hypertension; DM, diabetes mellitus; CVA, cerebrovascular accident; COPD, chronic obstructive pulmonary disease; AST, Aspartate aminotransferase; ALT, Alanine transaminase; LVAD, left ventricular assist device.

\begin{tabular}{|c|c|c|c|}
\hline Variable & All $(n=40)$ & pRVAD $(n=19)$ & $\operatorname{sRVAD}(n=21)$ \\
\hline \multicolumn{4}{|l|}{ Demographics } \\
\hline Age (years) & $58.6 \pm 11.8$ & $61.7 \pm 12.5$ & $55.7 \pm 10.6$ \\
\hline Gender (M) & $34(85.0 \%)$ & $17(89.5 \%)$ & $17(80.1 \%)$ \\
\hline BMI $\left(\mathrm{kg} / \mathrm{m}^{2}\right)$ & $29.0 \pm 6.2$ & $29.1 \pm 6.1$ & $28.9 \pm 6.3$ \\
\hline \multicolumn{4}{|l|}{ Comorbidities } \\
\hline CAD & $19(47.5 \%)$ & $10(52.6 \%)$ & $9(42.8 \%)$ \\
\hline HTN & $21(52.5 \%)$ & $10(52.6 \%)$ & $11(52.4 \%)$ \\
\hline DM & $13(30.3 \%)$ & $6(31.5 \%)$ & $7(33.3 \%)$ \\
\hline CVA & $3(7.5 \%)$ & $1(5.3 \%)$ & $2(9.5 \%)$ \\
\hline COPD & $4(10.0 \%)$ & $3(15.8 \%)$ & $1(4.8 \%)$ \\
\hline Smoker & $17(42.5 \%)$ & $9(47.4 \%)$ & $8(38.1 \%)$ \\
\hline \multicolumn{4}{|l|}{$\underline{\text { Lab Values }}$} \\
\hline Creatinine (mg/dL) & $1.6 \pm 0.6$ & $1.6 \pm 0.6$ & $1.6 \pm 0.6$ \\
\hline Lactate $(\mathrm{mmol} / \mathrm{L})$ & $2.8 \pm 1.8$ & $2.3 \pm 1.4$ & $3.5 \pm 2.2$ \\
\hline Hemoglobin (g/dL) & $9.3 \pm 1.3$ & $9.1 \pm 1.5$ & $9.4 \pm 1.2$ \\
\hline Total Bilirubin (mg/dL) & $2.0 \pm 1.3$ & $1.9 \pm 1.1$ & $2.2 \pm 1.5$ \\
\hline AST (U/L) & $140 \pm 271$ & $118 \pm 197$ & $160 \pm 330$ \\
\hline ALT (U/L) & $92 \pm 205$ & $55 \pm 107$ & $126 \pm 266$ \\
\hline \multicolumn{4}{|l|}{ Device } \\
\hline Impella RP & $4(13.3 \%)$ & $4(21.1 \%)$ & N/A \\
\hline Protek Duo & $15(37.5 \%)$ & $15(78.9 \%)$ & N/A \\
\hline Centrimag & $21(52.5 \%)$ & N/A & $21(100 \%)$ \\
\hline HeartMate II & $19(47.5 \%)$ & $5(26.3 \%)$ & $14(66.7 \%)$ \\
\hline HeartWare & $6(15.0 \%)$ & $1(5.3 \%)$ & $5(23.8 \%)$ \\
\hline HeartMate 3 & $14(35.0 \%)$ & $13(68.4 \%)$ & $1(4.8 \%)$ \\
\hline Other & $1(2.5 \%)$ & 0 & $1(4.8 \%)$ \\
\hline \multicolumn{4}{|l|}{$\underline{\text { Device Parameters }}$} \\
\hline Days post LVAD & $1(1-4.5)$ & $1(1-3)$ & $3(1-7)$ \\
\hline RVAD flow (L/hr) & $4.6 \pm 0.9$ & $4.0 \pm 0.4$ & $5.2 \pm 0.9$ \\
\hline LVAD flow (L/hr) & $5.2 \pm 1.3$ & $4.8 \pm 0.8$ & $5.8 \pm 1.7$ \\
\hline
\end{tabular}




\section{Table 2.}

Hemodynamic Parameters at time of device insertion for patients receiving percutaneous and surgical RVAD. All measurements reported were collected after implantation of durable left ventricular assist device and prior to implantation of the right ventricular assist device. pRVAD, percutaneous right ventricular assist device; sRVAD, surgical right ventricular assist device. CVP, central venous pressure; PA, pulmonary artery; MAP, mean arterial pressure; SVR, systemic vascular resistance; PVR, pulmonary vascular resistance; CO, cardiac output; CI, cardiac index; CPO, cardiac power output; CPI, cardiac power index; PAPI, pulmonary artery pulsatility index; RVSWI, right ventricular stroke work index.

\begin{tabular}{llll}
\hline Hemodynamic parameter (units, N) & All & pRVAD $(\mathbf{n = 1 9})$ & sRVAD (n=21) \\
\hline CVP (mmHg, 34) & $15.9 \pm 2.8$ & $15.6 \pm 2.4$ & $16.2 \pm 3.3$ \\
PA Systolic (mmHg, 29)) & $40.3 \pm 11.3$ & $41.5 \pm 12.3$ & $38.0 \pm 9.4$ \\
PA Diastolic (mmHg, 29)) & $22.3 \pm 5.1$ & $21.7 \pm 4.6$ & $23.4 \pm 5.9$ \\
PA Mean (mmHg, 29) & $28.1 \pm 6.6$ & $27.9 \pm 6.7$ & $28.4 \pm 6.5$ \\
MAP (mmHg, 37) & $73.8 \pm 11.8$ & $72.2 \pm 10.7$ & $75.4 \pm 13.0$ \\
SVR (dyne*s/cm $\left.{ }^{5}, 35\right)$ & $1006 \pm 506$ & $1032 \pm 463$ & $978 \pm 561$ \\
PVR (wood units, 23) & $2.3 \pm 1.4$ & $2.5 \pm 1.6$ & $1.9 \pm 0.7$ \\
CO (L/min, 29) & $5.0 \pm 2.0$ & $4.8 \pm 1.4$ & $5.4 \pm 2.7$ \\
CI (L/min/m², 29) & $2.4 \pm 0.8$ & $2.3 \pm 0.5$ & $2.6 \pm 1.1$ \\
CPO (Watt, 29) & $0.9 \pm 0.4$ & $0.8 \pm 0.1$ & $1.0 \pm 0.5$ \\
CPI (Watt/m², 29) & $0.4 \pm 0.2$ & $0.4 \pm 0.1$ & $0.5 \pm 0.2$ \\
PAPI (25) & $1.2 \pm 0.6$ & $1.4 \pm 0.6$ & $1.0 \pm 0.5$ \\
RVSWI (g/m²/beat, 22) & $3.4 \pm 2.2$ & $3.8 \pm 2.2$ & $2.8 \pm 2.1$ \\
\hline
\end{tabular}


Table 3.

Hemodynamic parameters of patients receiving percutaneous and surgical RVAD prior to device implantation, 12- and 24-hours post implantation, and 24 hours post device removal. All measurements reported prior to right ventricular assist device placement were collected after implantation of durable left ventricular assist. pRVAD, percutaneous right ventricular assist device; SRVAD, surgical right ventricular assist device.

\begin{tabular}{|c|c|c|c|c|c|}
\hline Parameter (units, N) & pRVAD (n=19) & p value & sRVAD $(n=21)$ & p value & pRVAD vs. sRVAD p value \\
\hline \multicolumn{6}{|c|}{ Central Venous Pressure $(\mathrm{mmHg}, 34)$} \\
\hline Pre & $15.6 \pm 2.3$ & & $16.2 \pm 3.3$ & & 0.56 \\
\hline 12 hour & $13.3 \pm 2.8$ & 0.008 & $10.6 \pm 3.4$ & $<0.001$ & 0.01 \\
\hline 24 hour & $12.3 \pm 3.2$ & $<0.001$ & $11.2 \pm 2.8$ & $<0.001$ & 0.29 \\
\hline post & $10.9 \pm 3.0$ & $<0.001$ & $11.2 \pm 4.2$ & 0.008 & 0.88 \\
\hline \multicolumn{6}{|c|}{ Mean Arterial Pressure $(\mathrm{mmHg}, 37)$} \\
\hline Pre & $72.2 \pm 10.6$ & & $75.4 \pm 12.9$ & & 0.42 \\
\hline 12 hour & $81.4 \pm 7.2$ & 0.004 & $82.4 \pm 9.6$ & 0.08 & 0.773 \\
\hline 24 hour & $77.7 \pm 6.9$ & 0.08 & $82.6 \pm 12.0$ & 0.10 & 0.15 \\
\hline post & $83.7 \pm 9.6$ & 0.003 & $78.3 \pm 10.3$ & 0.56 & 0.20 \\
\hline \multicolumn{6}{|c|}{ Systemic Vascular Resistance (dyne* $/ \mathrm{cm}^{5}, 35$ ) } \\
\hline Pre & $1032 \pm 463$ & & $978 \pm 561$ & & 0.75 \\
\hline 12 hour & $878 \pm 397$ & 0.29 & $775 \pm 403$ & 0.28 & 0.49 \\
\hline 24 hour & $832 \pm 336$ & 0.15 & $671 \pm 237$ & 0.08 & 0.15 \\
\hline post & $920 \pm 217$ & 0.45 & $959 \pm 196$ & 0.93 & 0.70 \\
\hline \multicolumn{6}{|c|}{$\underline{\text { Cardiac Output (L/min, 29) }}$} \\
\hline Pre & $4.7 \pm 1.4$ & & $5.4 \pm 2.7$ & & 0.40 \\
\hline 12 hour & $6.9 \pm 1.9$ & 0.001 & $7.3 \pm 3.6$ & 0.19 & 0.70 \\
\hline 24 hour & $7.1 \pm 1.5$ & $<0.001$ & $8.1 \pm 3.7$ & 0.07 & 0.31 \\
\hline post & $6.5 \pm 1.5$ & 0.006 & $6.7 \pm 0.8$ & 0.32 & 0.84 \\
\hline \multicolumn{6}{|c|}{$\underline{\text { Cardiac Index }\left(\mathrm{L} / \mathrm{min} / \mathrm{m}^{2}, 29\right)}$} \\
\hline Pre & $2.3 \pm 0.5$ & & $2.6 \pm 1.0$ & & 0.30 \\
\hline 12 hour & $3.4 \pm 0.9$ & $<0.001$ & $3.9 \pm 1.1$ & 0.02 & 0.27 \\
\hline 24 hour & $3.5 \pm 0.8$ & $<0.001$ & $4.6 \pm 1.3$ & $<0.001$ & 0.01 \\
\hline post & $3.3 \pm 0.6$ & $<0.001$ & $3.5 \pm 0.6$ & 0.11 & 0.45 \\
\hline \multicolumn{6}{|l|}{ Lactate (mg/dL 33) } \\
\hline Pre & $2.28 \pm 1.4$ & & $3.46 \pm 2.2$ & & 0.07 \\
\hline 12 hour & $1.83 \pm 1.5$ & 0.34 & $3.69 \pm 4.8$ & 0.87 & 0.12 \\
\hline 24 hour & $1.60 \pm 1.3$ & 0.13 & $2.59 \pm 3.6$ & 0.45 & 0.28 \\
\hline \multicolumn{6}{|c|}{ Vasopressors and Inotropes (number, 36) } \\
\hline Pre & $3.8 \pm 1.1$ & & $3.5 \pm 0.7$ & & 0.03 \\
\hline 12 hour & $3.2 \pm 0.9$ & 0.14 & $2.8 \pm 0.9$ & 0.02 & 0.07 \\
\hline 24 hour & $2.7 \pm 0.9$ & 0.03 & $2.4 \pm 0.9$ & $<0.001$ & 0.03 \\
\hline \multicolumn{6}{|l|}{ Creatinine $(\mathrm{mg} / \mathrm{dL}, 40)$} \\
\hline Pre & $1.63 \pm 0.6$ & & $1.57 \pm 0.6$ & & 0.77 \\
\hline 24 hour & $1.80 \pm 0.8$ & 0.41 & $1.53 \pm 0.6$ & 0.80 & 0.19 \\
\hline
\end{tabular}




\begin{tabular}{|c|c|c|c|c|c|}
\hline Parameter (units, N) & pRVAD $(n=19)$ & p value & $\operatorname{sRVAD}(n=21)$ & $p$ value & pRVAD vs. sRVAD p value \\
\hline 48 hour & $1.78 \pm 1.0$ & 0.57 & $1.46 \pm 0.6$ & 0.56 & 0.23 \\
\hline \multicolumn{6}{|c|}{ Urine Output $(\mathrm{mL} / 24 \mathrm{hr}, 39)$} \\
\hline Pre & $1700 \pm 1145$ & & $2175 \pm 2196$ & & 0.40 \\
\hline 24 hour & $2660 \pm 1783$ & 0.05 & $2647 \pm 2009$ & 0.48 & 0.98 \\
\hline 48 hour & $3081 \pm 3017$ & 0.07 & $2637 \pm 1745$ & 0.47 & 0.57 \\
\hline
\end{tabular}

ne Output (mL/24hr, 39) 
Table 4.

Outcomes with pRVAD and sRVAD. pRVAD, percutaneous right ventricular assist device; sRVAD, surgical right ventricular assist device; CVVH, continuous venovenous hemofiltration; HD, hemodialysis; PRBC, packed red blood cell; OHT, orthotopic heart transplant; ICU, intensive care unit.

\begin{tabular}{llll}
\hline Outcome & pRVAD $(\mathbf{n = 1 9 )}$ & sRVAD $(\mathbf{n = 2 1})$ & p value \\
\hline Days on Ventilator & $8(2,19)$ & $21(10,32)$ & 0.03 \\
Inhaled Nitric (hours) & $54(30,161)$ & $54.5(22.5,121.5)$ & 0.75 \\
CVVH/HD & $6(33.3 \%)$ & $9(42.8 \%)$ & 0.52 \\
PRBC Transfusions & $3(0,6)$ & $6.5(5,16)$ & $<0.001$ \\
OHT & $1(5.3 \%)$ & $3(14.3 \%)$ & 0.28 \\
Days in ICU (all) & $21(10,27)$ & $27(15,44)$ & 0.14 \\
Days in ICU (survivors) & $21(16,27)$ & $34(27,46)$ & 0.01 \\
Days in Hospital (all) & $34.5(21,59)$ & $60(21,95)$ & 0.35 \\
Days in Hospital (survivors) & $43.5(30,66)$ & $91(62,111)$ & 0.03 \\
Death at 30 days & $3(15.8 \%)$ & $7(33.3 \%)$ & 0.29 \\
In Hospital Death & $4(21.1 \%)$ & $9(42.9 \%)$ & 0.14 \\
Discharge Free from HD & $14(73.7 \%)$ & $10(47.6 \%)$ & 0.09 \\
\hline
\end{tabular}

